Abstract S7 Table 1 Utility values for patient and partner health states $(\mathrm{N}=104)^{\mathrm{a}}$

\begin{tabular}{llll}
\hline & & \multicolumn{2}{l}{ Utility Values $^{\mathbf{b}}$} \\
\cline { 3 - 4 } & & Mean & SD \\
\hline Patient health states & 1. No EDS & 0.93 & 0.11 \\
& 2. Mild EDS & 0.79 & 0.17 \\
& 3. Moderate EDS & 0.61 & 0.22 \\
4. Severe EDS & 0.55 & 0.24 \\
Partner health states & 5. No EDS & 0.95 & 0.08 \\
& 6. Mild EDS & 0.88 & 0.13 \\
& 7. Moderate EDS & 0.75 & 0.23 \\
& 8. Severe EDS & 0.67 & 0.26 \\
\hline
\end{tabular}

EDS, excessive daytime sleepiness; SD, standard deviation.

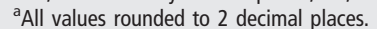

based on a scale of $0-1$, where 0 represents QoL equivalent to death and 1 represents the highest QoL.

This study aimed to elicit QoL values (utilities) from a societal perspective for patients with OSA with residual EDS of varying severity and, for the first time, for the partners of these patients, using a novel time trade-off (TTO) approach.

Methods Utility values for health states of varying severities of residual EDS in OSA were elicited using the TTO method in a study conducted with a United Kingdom general public sample $(\mathrm{N}=110)$. Four EDS severity health state descriptions were developed from both patient and partner perspectives (no EDS, mild EDS, moderate EDS, severe EDS). During face-toface interviews, participants were asked to 'trade off' time for each EDS health state for a shorter period in full health (010 years), first for the patient health states and then for the partner health states. TTO responses were converted to utility values (scale of $0-1$, where 0 represents $\mathrm{QoL}$ equivalent to death and 1 represents the highest QoL).

Results Mean utility scores declined with increasing EDS severity (table 1). In the context of the TTO exercise, participants were prepared to trade a mean of 4.5 life-years (out of a maximum of 10) to avoid the QoL consequences of severe EDS compared with best imaginable health and 3.3 life-years to avoid the QoL impact associated with being the partner of a patient with severe EDS.

Conclusions These results demonstrate the high potential impact of residual EDS on the QoL of patients with OSA and their partners. Estimated utility values for patients and partners declined with increased EDS severity. This is the first time that the impact of residual EDS in OSA on QoL and utility has been measured from the partner perspective, highlighting the range of potential benefits that could result from the use of novel treatment options (alongside primary airway therapy) for residual EDS.

Please refer to page A188 for declarations of interest related to this abstract.

\section{S8 THE IMPACT OF ARTEFACT-FREE RECORDING TIME ON THE DIAGNOSIS OF SLEEP DISORDERED BREATHING}

${ }^{1} \mathrm{AT}$ Knowles, ${ }^{2} \mathrm{H}$ Gajaweera, ${ }^{2} \mathrm{~J} \mathrm{Gavlak,}{ }^{1} \mathrm{CM}$ Hill, ${ }^{1} \mathrm{M}$ Stibalova, ${ }^{1} \mathrm{HM}$ Yuen, ${ }^{2} \mathrm{HJ}$ Evans. ${ }^{1}$ Faculty of Medicine, University of Southampton, Southampton, UK; ${ }^{2}$ Southampton Children's Hospital, University Hospital Southampton NHS Foundation Trust, Southampton, UK

10.1136/thorax-2021-BTSabstracts. 14
Background Sleep disordered breathing (SDB) is an umbrella term encompassing obstructive and central sleep apnoea. Overnight studies are used to confirm the diagnosis, however the minimum amount of artefact-free recording time (AFRT) required is yet to be determined in children.

Aim To determine the impact of different lengths of AFRT on the diagnosis of SDB.

Background Patients attended for either overnight cardiorespiratory polygraphy or polysomnography, alongside pulse oximetry sleep studies. Respiratory parameter reports were generated using the first 4, 5, 6 and 7 hours of AFRT. Clinically relevant cut-off (CRCO) values were used in conjunction with common diagnostic parameters: Obstructive AHI (OAHI; $\left.\mathrm{CRCO}^{32}\right)$; Central Apnoea-Hypopnoea Index (CAHI; $\left.\mathrm{CRCO}^{35}\right) ; 3 \%$ Oxygen Desaturation Index (ODI3\%; $\left.\mathrm{CRCO}^{3} 6\right) ; 4 \%$ Oxygen Desaturation Index (ODI4\%; $\left.\mathrm{CRCO}^{3} 4\right)$. Studies that effectively had diagnostic changes across different AFRTs, where diagnostic parameter values fluctuated above and below the CRCOs, were noted as 'Cases of Change' (COC). Receiver operating characteristic (ROC) curves determined ranges of parameter values at 4 hours to predict the presence of COC across the subsequent AFRTs.

Results 137 children (0.39-17.98 years) were consecutively recruited. The OAHI, CAHI, ODI3\% and ODI4\% had means of $1.54(\sigma=2.66), 1.56(\sigma=3.43), 5.21(\sigma=6.53)$ and $2.77(\sigma=4.42)$ respectively. Where children achieved 7 hours of AFRT, COC at 4 to 7 hours were seen as follows: $\mathrm{OAHI}^{32}=9.7 \%(10 / 103)$; $\mathrm{CAHI}^{3} 5=2.9 \%(3 / 103) ; \mathrm{ODI} 3 \%^{3} 6=3.7 \%$ (4/109); ODI $4 \%{ }^{3} 4$ $=1.8 \%(2 / 109)$. For the $\mathrm{OAHI}^{3} 2$, optimal points on ROC curves demonstrated a range with a lower limit of 0.875 $(\mathrm{AUC}=0.733 ; 50 \%$ sensitivity; 93\% specificity) and an upper limit of 3.125 (AUC $=0.968 ; 100 \%$ sensitivity; $81 \%$ specificity). Conclusion Studies with 4 hours AFRT are likely to yield diagnostic results in at least $90 \%$ of cases when commonly used cut-off criteria are applied. This study has identified ranges at 4 hours within which diagnostic change is most likely with longer periods of AFRT. Consideration should be given to repeating short studies where values at 4 hours lie within these ranges.

\section{S9 1 THE MANAGEMENT OF SLEEP DISORDERED BREATHING IN PEOPLE LIVING WITH HIV}

${ }^{1} \mathrm{~A}$ Saigal, ${ }^{1} \mathrm{~A}$ Jaffer, ${ }^{1} \mathrm{AJ}$ Shah, ${ }^{2} \mathrm{SK}$ Mansell, ${ }^{1} \mathrm{E}$ Pickett, ${ }^{5} \mathrm{C}$ Smith, ${ }^{3,4} \mathrm{R}$ Rakhit, ${ }^{1,4} \mathrm{M}$ Johnson,

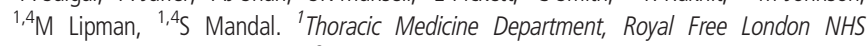
Foundation Trust, London, UK; ${ }^{2}$ Thoracic Medicine and Therapies Department, Royal Free London NHS Foundation Trust, London, UK; ${ }^{3}$ Cardiology Department, Royal Free London NHS Foundation Trust, London, UK; ${ }^{4}$ UCL Respiratory, London, UK; ${ }^{5}$ UCL, London, UK

\subsection{6/thorax-2021-BTSabstracts. 15}

Background Obstructive sleep apnoea (OSA) affects up to $24 \%$ of the general population and is associated with increased cardiovascular disease, cognitive impairment and depression. Continuous positive airway pressure (CPAP) is recommended for moderate-severe OSA although poor adherence affects treatment outcomes. We sought to identify OSA prevalence in people living with HIV (PLWH) and evaluate patient reported outcomes in those requiring CPAP.

Methods This observational study was conducted in an adult outpatient HIV clinic. We investigated through validated questionnaires and home sleep studies, OSA prevalence in PLWH reporting excessive daytime sleepiness (EDS), defined by an Epworth score $>10$. We evaluated symptom burden and CPAP outcomes. 
Abstract S9 Table 1 Demographics, baseline characteristics and summary of symptoms in PLWH reporting daytime somnolence with and without OSA

\begin{tabular}{|c|c|c|c|}
\hline & $\begin{array}{l}\text { Somnolent PLWH + no evidence of OSA } \\
(\mathrm{AHI}<5)\end{array}$ & $\begin{array}{l}\text { Somnolent PLWH + evidence of OSA } \\
(\mathrm{AHI} \geq 5)\end{array}$ & $P$ value \\
\hline N & 26 & 28 & \\
\hline Age (years) Mean \pm SD & $53.5 \pm 10.6$ & $53.4 \pm 10.8$ & 0.97 \\
\hline BMI (kg/m²) (Median IQR range) & $24(22.7-27.1)$ & $29(24.7-31.2)$ & 0.019 \\
\hline Gender (male) (\%) & $22 / 26(85)$ & $25 / 28(89)$ & 0.46 \\
\hline Current CD4 count & $617(233)$ & $559(372)$ & 1.0 \\
\hline \multicolumn{4}{|l|}{ Median (IQR range) } \\
\hline Antiretroviral therapy thought to contribute to insomnia (\%) & $7 / 28$ & $9 / 28$ & 0.258 \\
\hline Smoking status - Ex or current smokers (\%) & $17 / 26(65)$ & $15 / 28(54)$ & 0.42 \\
\hline Use of recreational drugs (\%) & $8 / 25(32)$ & $6 / 27(22)$ & 0.54 \\
\hline Pittsburgh Sleep Quality Index (scored 0 - 21) & $10(8)$ & $10.5(9)$ & 0.92 \\
\hline \multicolumn{4}{|l|}{ Median (IQR range) } \\
\hline Fatigue Severity Scale (scored $9-63$ ) & $46(20)$ & $39(28)$ & 0.35 \\
\hline \multicolumn{4}{|l|}{ Median (IQR range) } \\
\hline Generalised Anxiety Disorder - 7 (scored 0 - 21) & $11(13)$ & $6.5(9)$ & 0.16 \\
\hline \multicolumn{4}{|l|}{ Median (IQR range) } \\
\hline Patient Health Questionnaire- 9 Depression Score (Scored 0 - 27) & $14(12)$ & $10(9)$ & 0.50 \\
\hline \multicolumn{4}{|l|}{ Median (IQR range) } \\
\hline Standardised EuroQOL 5D-5L & $0.691(0.270)$ & $0.715(0.451)$ & 0.92 \\
\hline Median (IQR range) & & & \\
\hline
\end{tabular}

Results Between May 2018 - Nov 2019, we recruited 314 participants. $23.2 \%(n=73)$ reported EDS with significantly higher insomnia, fatigue, anxiety, depression scores and lower quality of life ( $p<0.0001$ for all variables compared to PLWH without EDS).

$54 / 73(73 \%)$ agreed to screening with a home sleep study; $52 \%(n=28 / 54)$ had sleep apnoea; moderate-severe in $57 \%$ (16/28). Patients with confirmed OSA had higher BMI (29 vs. $24 \mathrm{~kg} / \mathrm{m}^{2} ; \mathrm{p}=0.019$ ) but no differences in symptom burden, compared to those without OSA (table 1).

$31 \%(n=5)$ did not start or tolerate initial treatment. Of those using CPAP $(n=11)$, mean six week compliance was $6.22 \pm 2.16$ hours. Post-treatment AHI was 5.8/hr \pm 5.13, significantly improved from baseline $(37.8 \pm 22.48$; mean difference -31.98 (CI -46.5 to -17.45 ), $\mathrm{p}=0.001$ ). Mean 6 week Epworth score improved to $8.8 \pm 5.4$ from $15.8 \pm 3.96$; mean difference -7 (CI -12.1 to $-1.9, \mathrm{p}=0.014)$. By 3 months; further increase of 0.2 hours compliance (CI -1.1 to $1.5, \mathrm{p}=0.739$ ) was seen.

Conclusion There is unmet need in diagnosing and managing OSA in PLWH. Despite patients' significant symptom burden, barriers exist with engagement towards specialist care. Given that we demonstrate improved EDS in PLWH persevering with treatment, we need to find better approaches to support willingness towards investigation, treatment uptake for sleep disordered breathing and overall health management.

\section{REFERENCE}

1. Jaffer $A$, et al. Investigating excessive daytime sleepiness in adults living with HIV in the UK. Thorax. 2018:73(4).

\section{S10 AHI DOES NOT ADEQUATELY REFLECT OSA SEVERITY}

E Lombard, S Merritt. Sleep Disorder Unit, Conquest Hospital, East Sussex Healthcare NHS Trust, Hastings, UK

10.1136/thorax-2021-BTSabstracts. 16
Introduction Obstructive sleep apnoea (OSA) affects an estimated 1.5 million adults in the $\mathrm{UK}^{1}{ }^{1}$ resulting in significant cardiovascular consequences. Level of hypoxia and degree of sympathetic activation are postulated to play a role.

The Apnoea Hypopnoea Index (AHI) is used as a tool to assess severity of OSA. AHI counts the number of apnoeas and hypopnoeas per hour. It does not measure depth or duration of hypoxia and may underestimate the risk of complications. The aim of this study was to evaluate the relationship between AHI and the burden of hypoxia.

Method This was a retrospective study, using data from noxturnal sleep studies. Equal numbers of each OSA severity, defined by AHI, were selected consecutively from 122 adult patients who underwent sleep studies between Dec 2020 and May 2021. Demographic data, AHI and percentage time spent with oxygen saturations $<90 \% \quad(\%$ $\mathrm{T}<90 \%$ ) were recorded. Excel was used for analysis and Spearman's rank used to calculate the correlation coefficient (rho, r).

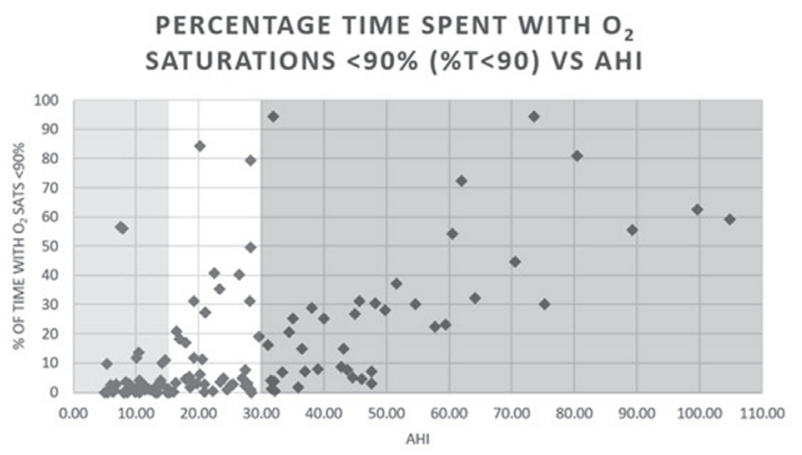

Abstract S10 Figure 1 Percentage time spent with $\mathrm{O}_{2}$ saturations $<90 \%(\% \mathrm{~T}<90)$ vs $\mathrm{AHI}$ 